Copyright ( 2008 IEEE. Reprinted from IEEE Transactions on Antennas and Propagation, 2008; 56 (5):1402-1412

This material is posted here with permission of the IEEE. Such permission of the IEEE does not in any way imply IEEE endorsement of any of the University of Adelaide's products or services. Internal or personal use of this material is permitted. However, permission to reprint/republish this material for advertising or promotional purposes or for creating new collective works for resale or redistribution must be obtained from the IEEE by writing to pubs-permissions@ieee.org.

By choosing to view this document, you agree to all provisions of the copyright laws protecting it. 


\title{
Markov Approach to Percolation Theory Based Propagation in Random Media
}

\author{
Tamath Rainsford, Member, IEEE, and Axel Bender
}

\begin{abstract}
For line-of-sight links in random media or urban areas, propagation may be approximated through sequential reflections of an optical ray in a two-dimensional medium of disordered lossless scatterers. Franceschetti et al. approximated such percolation-based optical-ray propagation by a Markov process with two absorbing barriers, provided numerical solutions for the probability of a ray passing through the percolation lattice and solved-both approximately and exactly - a corresponding problem based on the theory of martingales. In this paper we solve exactly the Markov-theoretical formulation of the problem and prove that both the Markov and martingale approaches are equivalent. Our proof is an application of the Perron-Frobenius theory which provides an elegant framework for the study of the asymptotic behavior of stochastic processes. We demonstrate that for a wide range of vacancies and incident angles the exact solution of the Markov-theoretical formulation performs significantly better than the commonly used Wald approximation in the martingale approach. This has a number of implications on the accuracy of the model, especially for low density propagation media.
\end{abstract}

Index Terms-Electromagnetic propagation in random media, Markov processes, martingales, mobile communication, urban areas.

\section{INTRODUCTION}

$\mathbf{T}$ HERE IS AN ever-increasing requirement for the high volume communication of information to homes and businesses. This information needs to be delivered speedily and with low latency. The volume of information to be delivered is escalating, as is the per-user capacity. The problem of how to deliver this information, from the provider to the customer, is known as the "Last Mile Problem." The problem is essentially about distribution, which is common to many other fields such as water distribution in irrigation systems and blood flow in biological systems. Wireless systems are an obvious solution because their losses are less than those of wired systems over the distances required. As wireless systems are unguided, they are subject to atmospheric losses and losses caused by obstructions such as buildings and terrain. On the other hand, for point-to-multi-point systems reflections caused by building blockage can be exploited. In general, interference of a signal due to reflections, refractions and diffractions need to be considered.

Manuscript received April 30, 2007; revised November 5, 2007.

T. Rainsford is with the School of Electrical and Electronic Engineering, University of Adelaide, Adelaide SA 5005, Australia (e-mail: tamath@eleceng.adelaide.edu.au).

A. Bender is with the Land Operations Division, Defence Science and Technology Organisation (DSTO), Australia, Edinburgh SA 5111, Australia (e-mail: axel.bender@defence.gov.au).

Digital Object Identifier 10.1109/TAP.2008.922626
While a full electromagnetic theory can be used to study the transmission characteristics of a signal, the equations are typically difficult to solve with many formulations being intractable. For this reason various approximations and alternative models for propagation have been developed, which include empirical (or statistical), semi-empirical and deterministic (or site-specific) approaches (see, for instance, [1]-[3] and references therein). Empirical models are based on the statistical characterization of the average attenuation of a transmitted signal and include the Okumura-based models developed in the COST 231 project [4], [5] and the Hata Model [6]. Most of the semi-empirical models are based on the COST 231 Walfisch-Ikegami model and take into account streets guiding effects and multi-screen diffraction effects [7], [8], [5]. Deterministic approaches such as ray-tracing techniques, image methods and the finite-difference time-domain model, typically have a physical basis, and require a vast amount of data regarding the geometry of the problem, the terrain profiles, the locations of building, trees and other scatterers and many more (see, e.g., [1] and references therein).

A relatively new deterministic model that is computationally inexpensive is the percolation-theory based approach in [9]. Franceschetti et al. consider a model of optical-ray propagation across a medium of disordered lossless scatterers. In this model the medium through which the ray propagates is treated as a two-dimensional percolating lattice. The idea is to determine the probability of penetration as a function of scatterer density and the ray's incident angle. This probability can be linked to the probability of the electromagnetic field being received [10]. In order to derive closed analytical expressions, Franceschetti et al. further describe the percolation of the ray stochastically. They use the theories of Markov chains and martingales in their analysis of the resulting random walk scenario. Thus, strictly speaking, they move away from percolation theory; however, they show that for scatterer densities below the percolation threshold and for incidence angles around $45^{\circ}$ the results obtained by employing probabilistic approximations are in very good agreement with numerical simulations of percolation-lattice based optical-ray propagation.

Percolation theory dates back as far as 1957 [11] and is still an active area of research. Percolation has been used to describe many very different physical phenomena, including forest fires [12], gelation of polymers [13] and semiconductors [14]. Good references on the theory can be found in the books of Kesten [15] and Stauffer [16]. Percolation theory is a very simple model for a disordered system. The random medium is defined through a set of regular lattice points that are either: site percolation, which uses the lattice vertices, or bond percolation, which uses lattice edges. In site percolation each site is occupied randomly with 
probability $p$ whereas in bond percolation $p$ defines the degree of connectivity. These occupied sites or bonds then represent certain physical properties. For example in the well-known diffusion model of forest fires [16] the occupied sites are trees. Occupied sites form clusters, which are simply groups of nearest neighbor sites. Percolation deals with these clusters. Important to percolation theory is the existence of a percolation threshold $p_{c}$. At values of $p>p_{c}$, for the first time there is an unbroken path from one side of the system to the other, i.e., there is a cluster that spans the lattice, and it is said that the lattice "percolates." In the forest fire algorithm, for instance, a fire is started on one side of the lattice and propagates toward the other side. The fire is only able to propagate through combustible cells of the lattice. It is then easy to determine if a cluster spans the lattice-it does if there are any cells in the last row of the lattice that are "burnt." The percolation threshold $p=p_{c}$ defines a phase transition at which the behavior of a system changes.

The Franceschetti lattice [9] is an infinite two-dimensional regular square lattice and each cell is either occupied with a scatterer or not. Under the assumption that the occupancy status of a cell is random and independent of any other cell in the lattice one can define an occupancy probability density $q=1-p$, i.e., a cell is "empty" or unoccupied with the probability $p$. Only values of $p$ above $p_{c} \approx 0.59275$ are of interest for the Last Mile Problem, as it is for these probability densities that optical-ray percolation can occur, that is, an optical ray can propagate from one side of the lattice to the other. If a cell is occupied the impinging ray is totally reflected; if, on the other hand, the cell is unoccupied then the ray progresses freely and without any loss. Scatterers are impenetrable and diffraction at the edges of the lattice is ignored. The fact that the effect of refraction and diffraction on transmission is ignored limits the application of the model. Furthermore, the model is two-dimensional and based on the premise that a site being empty or occupied is statistically independent on neighboring site states, i.e., there is no structural organization. As cities usually have a fairly high degree of structure the assumptions of two-dimensionality and statistical independence reduce the applicability of the approach with respect to modelling propagation in urban areas. Nevertheless, the Franceschetti model provides a simple foundation for examining how a city topology can modify certain propagation characteristics, such as losses in power densities. It may also prove useful, if appropriately extended, in solving some specific problems related to line-of-sight (LOS) links in random media or urban areas.

Reference [17] improves the basic model by generalisation to a non-uniform distribution of empty cells. Here site states in a lattice row $j$ are described through a more general occupation profile $q_{j}$, and it is demonstrated that the Markov approach to solving the percolation lattice problem is very accurate for random percolation lattices with $p>p_{c}$. Thus it appears that finding an exact (and potentially simple) analytical solution of the Markovian formulation of optical-ray propagation in random media [9] would help advance the field and facilitate future research in this area.

In [9] an approximate and widely used expression for propagation depth is based on describing a sequence of reflections, or "jumps," of the optical ray in the percolation lattice as a martingale and then employing Wald's approximation when calcu- lating conditioned ensemble averages that relate to optical rays penetrating certain lattice levels. Franceschetti et al. see a need for additional approximations, such as the Wald approximation, because they found that the exact solution of the Markov approach " $[\cdots]$ requires numerical manipulations of the transition matrix $[\cdots]$ that do not appear to lend themselves to a simple analytical expression." In this paper, we actually derive an exact analytical solution for the Markov approach to optical-ray propagation in random media by manipulating the Markov transition matrix and employing the Perron-Frobenius theory for non-negative matrices (see [18] for a description of the theory). The solution turns out to be of great simplicity, and we shall prove that it is equal to the exact solution of the martingale approach. Unfortunately, this exact solution appears to have been forgotten by parts of the research community as in some work following [9] only the (inaccurate) Wald approximation is used [17], [19]. Thus we believe that the derivation we present in this paper will be a useful addition to the body of work on percolation-theory based optical-ray propagation.

Our paper is organized in the following way. In Section II we define in more detail the mathematical problem that we will solve, and introduce our notation. In Section III we describe how the Markov chain approach can be applied to ray propagation in random media and we formulate the problem in the limit of infinitely many reflections to recover the model of G. Franceschetti et al. in [9]. We then highlight that in this limit the Markov chain approach has to be equivalent to an approach that solves the stochastic ray propagation process through employing the theory of martingales. We provide a proof of this observation by deriving an exact solution of the Markov approach. In Section IV we interpret our result in the context of the Perron-Frobenius theory, which provides a general and elegant framework for determining the asymptotic behavior of Markov processes. We also critically assess the accuracy of the Wald approximation to the martingale-based approach of [9], [17] and provide a physical interpretation of our main result. In Section $\mathrm{V}$ we conclude.

\section{Propagation Model}

The lattice is a simple two-dimensional square lattice that is infinite in both its horizontal and vertical extensions. Sites are occupied independently of neighboring sites with a site occupation of $q=1-p$ giving a completely random distribution of occupancies. Moreover, $p>p_{c}$ so that the lattice is percolating. The medium is assumed lossless (scatterers are impenetrable) and diffraction effects are ignored. Fig. 1 shows an example of such a percolating lattice and a ray propagating in the lattice. The $k$ levels of the lattice are labelled with indices with level $i=0$ being just outside the lattice and $i=1$ the first row of the lattice. An impinging ray has angle of incidence $\theta$ and first has to enter the lattice before propagating within it.

In [9] the problem is divided into two parts: first finding the probabilities, $Q_{0}(j)$, for the ray entering the lattice and being reflected at lattice level $j$ for the first time; then formulating the probability distributions for successive jumps. Geometrical ray optics allows for the determination of the ray's path based on only the incidence angle and the initial entry point into the lattice. Of course, the real problem is to determine the propagation 


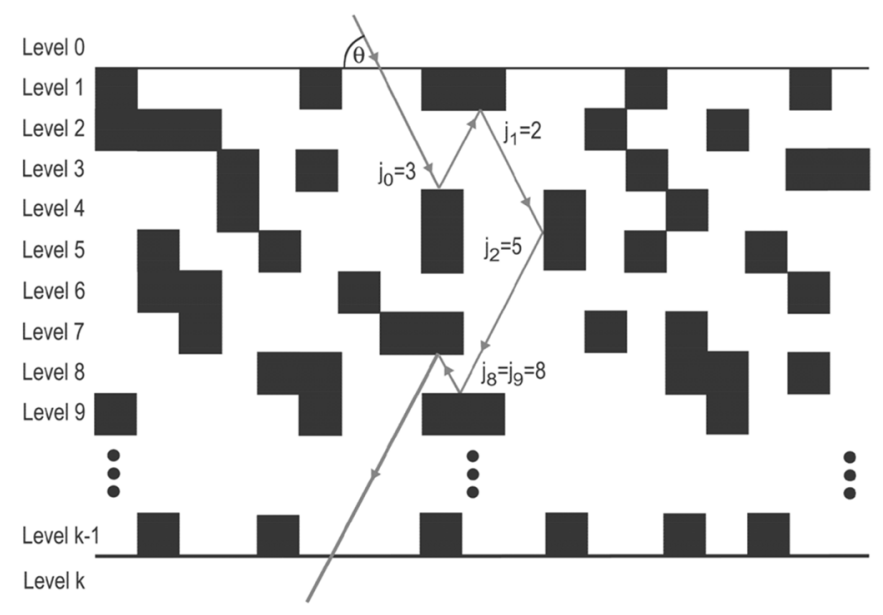

Fig. 1. Example of a ray propagating through a percolating lattice.

of a large number of impinging rays. Thus we assume a statistical number of rays (which is equivalent to saying that the electromagnetic wave is a parallel plane wave), and hence the entry point dependence disappears and can be replaced by an entry probability, which in the case of the percolation lattice is equal to $p$.

In Fig. 1 it is shown that an impinging ray, upon entering the lattice, could propagate freely through a number of layers since the first reflection does not necessarily occur at the first or even the second level. In order to calculate the probability for a ray being reflected at level $i$ we note that the number of vertical and horizontal level faces that need to be crossed freely prior to the reflection at level $i$ is $(i-1)(1+\tan \theta)+1$ [9]. This leads to some new definitions: the effective probability of a site to be empty:

$$
p_{e}=p^{1+\tan \theta}
$$

and the effective probability of a site to be occupied

$$
q_{e}=1-p_{e} .
$$

It then can be shown that the probability for the first ray reflection occurring at level $i$ of the lattice is given by [9]

$$
Q_{0}(i)= \begin{cases}q, & \text { for } i=0 \\ p p_{e}^{i-1} q_{e}, & \text { for } 0<i<k \\ \sum_{j=k}^{\infty} p p_{e}^{i-1} q_{e}=p p_{e}^{k-1}, & \text { for } i=k\end{cases}
$$

Within the lattice a ray may be reflected such that it remains within the lattice row, "jumps" forward or "jumps" backward. These cases are dependent on whether the reflection takes place on a horizontal face or a vertical face. The latter results in a jump forward while the former describes a jump backward. For a ray entering the lattice obliquely the probabilities of hitting a horizontal face, $\xi_{h}$, or a vertical face $\xi_{v}$ are not equal for incidence angles that differ from $45^{\circ}$

$$
\xi_{h}=\frac{1}{1+\tan \theta} \text { and } \xi_{v}=1-\xi_{h} .
$$

As argued in [9] the overall probability for a ray heading in the forward direction after $n$ reflection is given by $\alpha_{n} p_{e}$

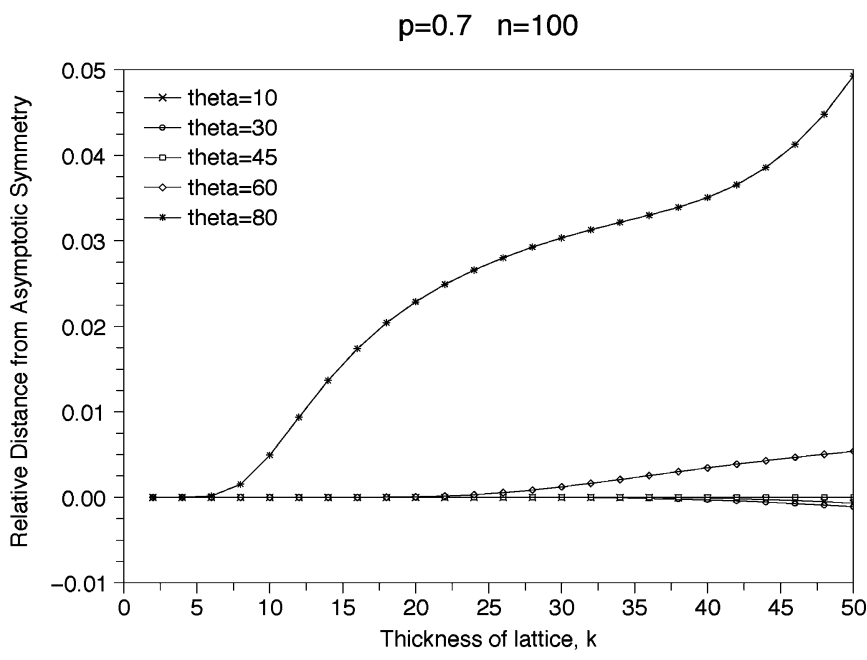

Fig. 2. Measure of how well, after $n=100$ jumps, ray propagation in percolation lattices can be described by symmetric jump distributions, e.g., a measure of 0.01 ( 0.02 , etc.) describes a $1 \%$ ( $2 \%$, etc.) deviation from asymptotic symmetry. Here,$p=0.7$ and $\theta=10^{\circ}, 30^{\circ}, 45^{\circ}, 60^{\circ}$ and $80^{\circ}$.

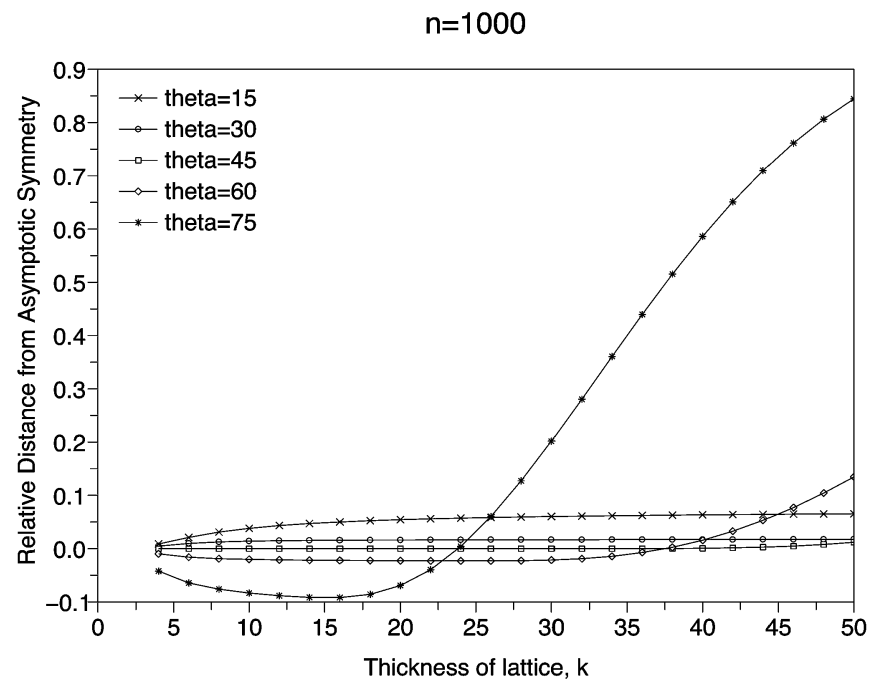

Fig. 3. Same as Fig. 3 but for $n=1000$ reflections.

and the probability for backward propagation is $\left(1-\alpha_{n}\right) p_{e}$, where $\alpha_{n}=(1 / 2)-(1 / 2)\left(\xi_{v}-\xi_{h}\right)^{n}$. Except for $\theta=0^{\circ}$ and $90^{\circ} \lim _{n \rightarrow \infty} \alpha_{n}=(1 / 2)$, i.e., in the limit of infinitely many reflections forward and backward jumps become equally likely. This fact indicates that the distribution of jumps is asymptotically symmetric irrespective of the angle of incidence (with the exception of exactly horizontal and vertical rays). Figs. 2 and 3 illustrate that for $p=0.7$ and incidence angles between $10^{\circ}$ and $80^{\circ}$ asymptotic symmetry is reached with $5 \%$ accuracy after 100 reflections and $1.8 \%$ after 1000 reflections in thin lattices $(k \leq 50)$. The shown measure of relative distance from asymptotic symmetry is evaluated by employing the inhomogeneous Markov approach to optical-ray propagation in random percolation lattices in which incidence-angle dependence is exactly accounted for. This approach is described in more detail in [20].

In [9], Franceschetti et al. observe that under the assumption that backward and forward jumps have equal probability, the 
stochastic process of ray propagation can be considered a martingale. Employing the theory of martingales (see, e.g., [21]) the authors determine the probability, $Q_{n}(k)$, for reaching the depth $k$ after $n$ successive reflections. We will argue in Section III-C that the theory of martingales must lead to exactly the same solution for $\lim _{n \rightarrow \infty} Q_{n}(k)$ as a Markov theoretical approach in which the jumps are assumed to be independently identically distributed (iid), i.e., $\alpha_{n} \rightarrow(1 / 2) \forall n$. We will prove this argument by solving analytically the Markov theoretical approach and calculating $\vec{Q}_{\infty}$, thus complementing [9]. ${ }^{1}$

\section{MARKov ApProAch to RAY PRopagation IN THE Limit OF IID JUMPS}

\section{A. Definition of Markov Chain}

When formulating ray propagation in a two-dimensional random medium as a Markov chain problem, we make the basic assumption that jumps between different lattice layers are independent of the ray's history of previous reflections. This assumption can be articulated as follows: given the probability $p_{j_{1} j_{2}}$ that a reflection at layer $j_{2}$ occurs under the condition of a reflection at layer $j_{1}$, i.e., $p_{j_{1}} j_{2}$ describes the probability of a "jump" from $j_{1}$ to $j_{2}$; and given the probability $Q_{0}\left(j_{0}\right)$ that the ray initially propagates to lattice layer $j_{0}$ prior to its first reflection, then the probability $Q_{n}\left(j_{0}, j_{1}, \ldots, j_{n}\right)$ of a jump sequence from layer $j_{0}$ to $j_{n}$ via layers $j_{1}, j_{2}, \ldots, j_{n-1}$ is defined by [22]

$$
Q_{n}\left(j_{0}, j_{1}, \ldots, j_{n}\right)=Q_{0}\left(j_{0}\right) p_{j_{0} j_{1}} p_{j_{1} j_{2}} \ldots p_{j_{n-1} j_{n}}
$$

Summing over all possible states $j_{0}, j_{1}, j_{2}, \ldots, j_{n-1}$ we get the probability $Q_{n}\left(j_{n}\right)$ of the ray reaching layer $j_{n}$ after (exactly) $n$ reflections

$$
Q_{n}\left(j_{n}\right)=\sum_{j_{0}, j_{1}, \ldots j_{m-1}=0}^{k} Q_{0}\left(j_{0}\right) p_{j_{0} j_{1}} p_{j_{1} j_{2}} \ldots p_{j_{n-1} j_{n}}
$$

By defining a $(k+1) \times(k+1)$ matrix $M$ with components $M(i, j) \equiv p_{i j}$, (3) can be recast in the vector equation

$$
\vec{Q}_{n}^{T} \equiv \vec{Q}_{0}^{T} M_{k}^{n}
$$

with the last component of this vector, $Q_{n}(k)$, describing the probability of the ray passing through the percolation lattice or, more precisely, its $k$ th layer after $n$ reflections.

\section{B. Limit of Infinitely Many Reflections}

In [9] the probabilities $p_{i j}$ have been determined for the case in which the ray gets infinitely often reflected inside the lattice before either being reflected back $\left(j_{\infty}=0\right)$ or passing through the lattice $\left(j_{\infty}=k\right)$. In this case, the problem of solving (4) reduces to

$$
\vec{Q}_{\infty}^{T} \equiv \lim _{n \rightarrow \infty} \vec{Q}_{n}^{T}=\vec{Q}_{0}^{T} \lim _{n \rightarrow \infty} M_{k}^{n}
$$

${ }^{1}$ The vector $\vec{Q}_{\infty}$ has $k+1$ components; in our notation the components $a(i)$ of any given vector $\vec{a}$ range from $i=0$ to $i=k$. Similarly, we use $A(i, j)$ to denote a matrix element with both row index $i$ and column index $j$ ranging from 0 to $k$.
Under the assumption of asymptotic symmetry at every ray reflection (even the very first one)

$$
\alpha_{n} \approx \frac{1}{2}
$$

the matrix $M_{k}$ is given by [9]

$$
M_{k} \equiv \frac{1}{2}\left(\begin{array}{ccccc}
2 & 0 & \ldots & 0 & 0 \\
p_{e} & & & & p_{e}^{k-1} \\
p_{e}^{2} & & & & p_{e}^{k-2} \\
& & 2 X_{k} & & \\
\vdots & & & & \vdots \\
p_{e}^{k-1} & & & & p_{e} \\
0 & 0 & \ldots & 0 & 2
\end{array}\right)
$$

The matrix $X_{k}=\left(q_{e} / 2\right) \tilde{X}_{k}$ is a $(k-1)$ th minor of $M_{k}$ and is a symmetrical $(k-1) \times(k-1)$ matrix, $X_{k}^{T}=X_{k}$, with

$$
\tilde{X}_{k} \equiv\left(\begin{array}{ccccc}
2 & p_{e} & p_{e}^{2} & \cdots & p_{e}^{k-2} \\
p_{e} & 2 & p_{e} & \cdots & p_{e}^{k-3} \\
p_{e}^{2} & p_{e} & 2 & \cdots & p_{e}^{k-4} \\
\vdots & \vdots & \vdots & \ddots & \vdots \\
p_{e}^{k-2} & p_{e}^{k-3} & p_{e}^{k-4} & \cdots & 2
\end{array}\right)
$$

\section{IID Assumption and Absolute Fairness}

By imposing the condition of asymptotic symmetry, (6), at each reflection the ray has equal probability to be reflected forwards or backwards, irrespective of the angle of incidence. In probability theoretical terms this implies that the sequence of jumps $\left\{j_{n}\right\}$ is absolutely fair [23], i.e., the expectation value for jump $j_{n+1}$ is zero irrespective of the previous sequence of jumps $\left\{j_{i}\right\}$. Because the ray "loses the memory of previous reflections" whenever the next reflection occurs, the sequence of reflection layers $\left\{i_{n}\right\}$ is a martingale: if the $n$th reflection occurs at layer $i_{n}$ then the expectation value for the next layer of reflection is $i_{n}$ as well, i.e., the ensemble average $\bar{i}_{n}$ over all possibilities for the next jump $\left(j_{n+1}\right)$ is $i_{n}$. Therefore the random walk described by $M_{k}^{n}$ with $M_{k}$ defined in (7) is a martingale. If the martingale formulation is solved exactly (as done in [9]) then both martingale and Markov approaches have to lead to the same result. In the next subsection we will prove the correctness of this observation by showing that the exact solution of (5) is identical to the exact solution of the martingale approach as derived in [9].

We emphasize that the equivalence of the two approaches is only true under the simplifying assumption of iid jumps, (6). This condition is only exactly fulfilled when the incidence angle equals $\theta=45^{\circ}$. In [20] we discuss how the probabilistic approach to percolation-theory based ray propagation can be formulated such that incidence angles $\theta \neq 45^{\circ}$ are treated exactly. This leads to a generalized Markov formulation of the problem.

\section{Closed Analytical Solution of the Markov Approach}

In order to solve (5), we make use of spectral decomposition. Let $\Lambda_{k} \equiv\left(\vec{\omega}_{0}, \vec{\omega}_{1} \ldots, \vec{\omega}_{k}\right)$ be a matrix whose columns contain 
the right eigenvectors $\vec{\omega}_{i}, i \in\{0,1, \ldots, k\}$, of $M_{k}$, i.e.,

$$
M_{k} \Lambda_{k}=\Lambda_{k} \operatorname{diag}\left(\lambda_{0}, \lambda_{1}, \ldots, \lambda_{k}\right) .
$$

Then $M_{k}=\Lambda_{k} \operatorname{diag}\left(\lambda_{0}, \lambda_{1}, \ldots, \lambda_{k}\right) \Lambda_{k}^{-1}$ and thus $M_{k}^{n}=$ $\Lambda_{k} \operatorname{diag}\left(\lambda_{0}^{n}, \lambda_{1}^{n}, \ldots, \lambda_{k}^{n}\right) \Lambda_{k}^{-1}$, i.e.,

$\lim _{n \rightarrow \infty} M_{k}^{n}=\Lambda_{k} \operatorname{diag}\left(\lim _{n \rightarrow \infty} \lambda_{0}^{n}, \lim _{n \rightarrow \infty} \lambda_{1}^{n}, \ldots, \lim _{n \rightarrow \infty} \lambda_{k}^{n}\right) \Lambda_{k}^{-1}$.

The eigenvalues of $M_{k}$ can be evaluated from the characteristic equation $\operatorname{det}\left(M_{k}-\lambda I_{k+1}\right)=0 .^{2}$ By expanding the characteristic equation in cofactors we get $\operatorname{det}\left(M_{k}-\lambda I_{k+1}\right)=$ $(1-\lambda)^{2} \operatorname{det}\left(X_{k}-\lambda I_{k-1}\right)$, i.e., $\lambda_{0}=\lambda_{k}=1$ are two of the $k+1$ eigenvalues. ${ }^{3}$ The remaining $k-1$ eigenvalues of $M_{k}$ are identical to those of the matrix $X_{k}$ : the conditions $\Lambda_{k}(i, 0)=$ $\Lambda_{k}(i, k)=0$ define a subspace in which the eigenvectors and eigenvalues of $M_{k}$ are determined by the eigenvalues and eigenvectors of $X_{k}$.

According to the Frobenius theorem the largest eigenvalue of a Markov matrix $M$ is 1 . The theorem also states that there are exactly $r$ independent eigenvectors of eigenvalue 1 where $r$ denotes the number of recurrent classes of the Markov chain $M$; and the amplitudes of all other eigenvalues are smaller than $1.4^{4}$ The process described by (7) is a Markov chain which, for $p \neq 0$, has two ergodic (i.e., aperiodic recurrent) classes and one transient class, see also Section IV-A. Thus there are only two eigenvectors of eigenvalue 1 and the absolute value of all other eigenvalues is smaller than $1:\left|\lambda_{i}\right|<1^{5}$, i.e., $\lim _{n \rightarrow \infty}\left|\lambda_{i}\right|_{p \neq 0}^{n}=$ $0 \forall i \in\{1, \ldots, k-1\}$.

Hence

$$
\begin{aligned}
\lim _{n \rightarrow \infty} M_{k}^{n} & =\Lambda_{k} \operatorname{diag}(1,0,0, \ldots, 0,1) \Lambda_{k}^{-1} \\
& =\left(\vec{\omega}_{0}, \overrightarrow{0}, \ldots, \overrightarrow{0}, \vec{\omega}_{k}\right) \Lambda_{k}^{-1}
\end{aligned}
$$

and in order to solve (5) we therefore only need to calculate the two eigenvectors $\vec{\omega}_{0}$ and $\vec{\omega}_{k}$ and the first and last row of the inverse of $\Lambda_{k}$.

\footnotetext{
${ }^{2} I_{l}$ denotes the $l \times l$ identity matrix.

${ }^{3}$ The indices for these two eigenvalues have been arbitrarily chosen to be 0 and $k$.

${ }^{4}$ For an overview of the application of Perron-Frobenius theory to Markov processes see, e.g., [21]

${ }^{5}$ The case $p=0$ leads to the trivial problem $\lim _{p \rightarrow 0} M_{k}=I_{k+1}$, i.e., all sites of the percolation lattice are occupied. Thus a jump from layer $i$ to $j$ is impossible if $i \neq j$. As a result, with probability $q=1$ the ray gets reflected at the first layer of the lattice.
}

As detailed in Appendix A the two normalized eigenvectors can be obtained by solving the equation $M_{k} \vec{\omega}_{0 \mid k}=\vec{\omega}_{0 \mid k}$. They are (26)

$$
\vec{\omega}_{0}^{T}=\frac{1}{\sqrt{k+1}}(1,1, \ldots, 1)
$$

and (35)

$$
\vec{\omega}_{k}=Z^{-1}\left(\begin{array}{c}
2+q_{e}(k-2) \\
q_{e}(k-2) \\
q_{e}(k-4) \\
\vdots \\
q_{e}(k-2(k-1)) \\
-2-q_{e}(k-2)
\end{array}\right)
$$

with the normalization constant $Z$ given in (36).

The first row vector of $\Lambda_{k}^{-1}$ is

$$
\vec{\beta}_{0}^{T}=\frac{1}{2} \sqrt{k+1}(1,0,0, \ldots, 0,1)
$$

and the last row vector is

$$
\vec{\beta}_{k}^{T}=\frac{Z}{2\left(2 p_{e}+k q_{e}\right)}(1,0,0, \ldots, 0,-1)
$$

see Appendix B.

Inserting $\vec{\omega}_{0}, \vec{\omega}_{k}, \vec{\beta}_{0}$ and $\vec{\beta}_{k}$ into (11) leads to (12), shown at the bottom of the page, and thus (5) reads

$$
\vec{Q}_{\infty}^{T}=\left(1-p \frac{2 p_{e}+q_{e}}{2 p_{e}+k q_{e}}, 0, \ldots, 0, p \frac{2 p_{e}+q_{e}}{2 p_{e}+k q_{e}}\right)
$$

see Appendix $\mathrm{C}$ for details of the derivation.

Equation (13) is the exact solution of the Markov chain theoretical approach in the limit of infinitely many reflections $n \rightarrow$ $\infty$. An example of how with increasing number of reflections, $n$, the penetration probability $Q_{n}(k)$ asymptotically approaches (13) is depicted in Fig. 4.

\section{RESUlTS AND DISCUSSION}

\section{A. Perron-Frobenius Theory}

The approach used to derive the solution for $\lim _{n \rightarrow \infty} M_{k}^{n}$ in (12) is an application of the Perron-Frobenius theory for non-negative matrices. Through employment of both the Perron and Frobenius theorems it can be shown (see, e.g., [21]) that a Markov chain $M$ with $r$ recurrent classes and one or more transient classes:

$$
\lim _{n \rightarrow \infty} M_{k}^{n}=\frac{1}{2\left(2 p_{e}+k q_{e}\right)}\left(\begin{array}{ccccc}
2\left(2 p_{e}+k q_{e}\right) & 0 & \cdots & 0 & 0 \\
p_{e}+(k-1) q_{e} & 0 & \cdots & 0 & p_{e}+q_{e} \\
p_{e}+(k-2) q_{e} & 0 & \cdots & 0 & p_{e}+2 q_{e} \\
\vdots & \vdots & \ddots & \vdots & \vdots \\
p_{e}+q_{e} & 0 & \cdots & 0 & p_{e}+(k-1) q_{e} \\
0 & 0 & \cdots & 0 & 2\left(2 p_{e}+k q_{e}\right)
\end{array}\right)
$$




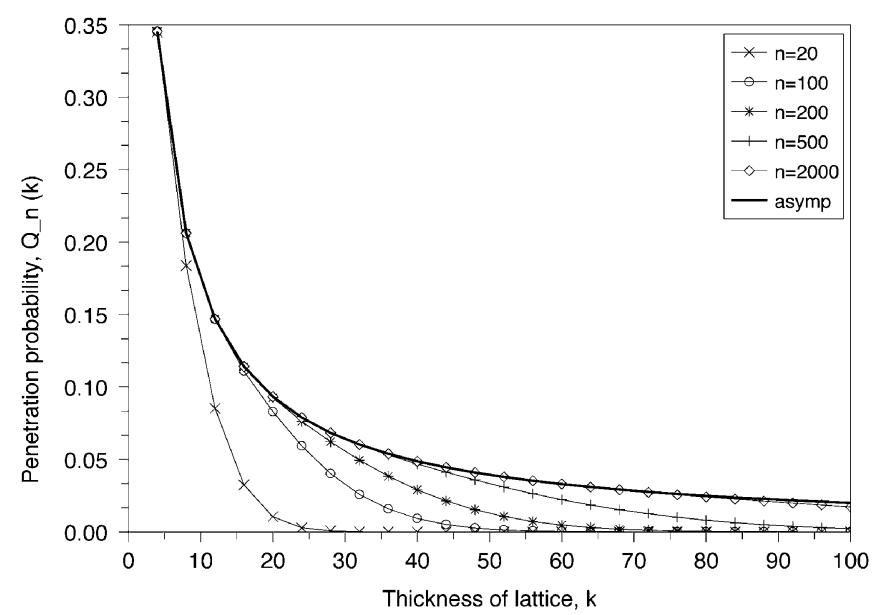

Fig. 4. $Q_{n}(k)$ as a function of lattice thickness $k$ for $p=0.7$ and $\theta=45^{\circ}$. The solid black curve shows the result for $n \rightarrow \infty$ as calculated from (13).

1) has exactly $r$ linearly independent left eigenvectors $\vec{\pi}_{1}^{T}, \ldots \vec{\pi}_{r}^{T}$ of eigenvalue $\lambda=1$ where $\vec{\pi}_{i}^{T}$ can be chosen to be a probability vector with positive components on the $j$ th recurrent class and zero elsewhere;

2) has exactly $r$ linearly independent right eigenvectors $\vec{\rho}_{1}, \ldots \vec{\rho}_{r}$ of eigenvalue 1 where $\vec{\rho}_{j}$ can be chosen to be a vector with the component $\rho_{j}(i)$ equal to the probability that the $j$ th recurrent class will ever be entered starting from state $i$

3)

$$
\lim _{n \rightarrow \infty} M^{n}=\sum_{i=1}^{r} \vec{\rho}_{i} \vec{\pi}_{i}^{T}
$$

The probabilistic interpretation of the percolation-theory based description of ray propagation in random media leads, under the iid assumption 6 , to the Markov process $M_{k}$ in (7) which has two recurrent classes, namely respectively the lattice levels 0 and $k$. The other lattice levels belong to the same transient class because:

1) all levels $i, j \in\{1, \ldots, k-1\}$ are connected with each other, which means that both transitions $i \rightarrow j$ and $j \rightarrow i$ are possible;

2) once a transition $i \rightarrow 0$ or $i \rightarrow k$ occurs, there is no return back to $i$. After all, the two levels 0 and $k$ are absorbing states.

Because of $r=2$ with each of the recurrent classes containing one state, we have $\vec{\pi}_{1}^{T}=\vec{e}_{0}^{T}$, and $\vec{\pi}_{2}=e_{k}^{T}$. This implies that $\vec{\rho}_{1}$ and $\vec{\rho}_{2}$ are respectively the first and last column vectors in $M_{k}^{\infty}$, (12)

$$
\begin{aligned}
\vec{\rho}_{1} & =\beta_{00} \vec{\omega}_{0}+\beta_{k 0} \vec{\omega}_{k} \\
& =\left(1,1-\gamma_{1}, 1-\gamma_{2}, \ldots, 1-\gamma_{k-1}, 0\right)^{T} \\
\vec{\rho}_{2} & =\beta_{0 k} \vec{\omega}_{0}+\beta_{k k} \vec{\omega}_{k} \\
& =\left(0, \gamma_{1}, \gamma_{2}, \ldots, \gamma_{k-1}, 1\right)^{T}
\end{aligned}
$$

with $\beta_{00}, \beta_{0 k}, \beta_{k 0}$ and $\beta_{k k}$ given in (39) and (40), and

$$
\gamma_{i}=\frac{p_{e}+i q_{e}}{2 p_{e}+k q_{e}} .
$$

Thus, according to the Perron-Frobenius theory, the matrix components $M_{k}^{\infty}(i, j)$ with $j \in\{0, k\}$ are the probabilities $\operatorname{Pr}\left(j_{\infty}=j \mid j_{0}=i\right)$ that (recurrent) lattice level 0 or $k$ will ever be entered starting from level $i$. A corollary of this interpretation is that $\vec{\rho}_{1}+\vec{\rho}_{2}=(1,1, \ldots, 1)^{T}$ : in the asymptotic limit of infinitely many reflections, irrespective of where a ray starts it is certain that it will end up in either of the two absorbing states, i.e., in either the 0th or the $k$ th level of the lattice.

\section{B. Comments on the Equivalence of Markov and Martingale Approaches}

The right eigenvector $\vec{\rho}_{2}$ in (16) is the same vector of probabilities $\operatorname{Pr}\left(j_{\infty} \geq k \mid j_{0}=i\right)$ that Franceschetti et al. derived by employing the theory of martingales exactly (see (41) in [9]). Equally, the expressions for $\vec{Q}_{\infty}$ are identical in both the Markov and the martingale approaches (i.e., our solution 13 is exactly the same as (42) in [9]). In relation to their results Franceschetti et al. note: "It is remarkable that, from a numerical point of view, relationship (42) is practically coincident for all values of $p$ and $\theta$ of interest, with the exact solution [of the Markov approach ....] Hence, it can be considered the right (non-approximate) expression of the propagation depth." Our observation in Section III.C and our derivation of expressions 13 and 16 show, that the described "coincidence" is no accident: The martingale and Markov approaches are merely two different ways of solving the same problem.

Unfortunately, since the publication of [9] the correct solution of the percolation theoretical approach to ray propagation for iid jumps appears to have been forgotten by some researchers. For instance, in [24], [19], [17] the 1999 paper [9] is cited but (13) is not referred to. Instead Wald's approximation to the martingale approach is used for further investigation of the percolation lattice problem. This approximation leads to the following expression for the probability vector $\vec{Q}_{\infty}$ :

$$
\left(\vec{Q}_{\infty}^{T}\right)_{\text {Wald }}=\left(1-\frac{p}{k q_{e}}\left(1-p_{e}^{k}\right), 0, \ldots, 0, \frac{p}{k q_{e}}\left(1-p_{e}^{k}\right)\right) .
$$

The relative error made when using approximation 18 rather than the exact solution 13 can be quantified by defining

$$
\operatorname{Err} \equiv 1-\frac{\left(Q_{\infty}(k)\right)_{\mathrm{Wald}}}{Q_{\infty}(k)}
$$

Figs. 5 and 6 depict this quantity respectively as a function of probability $p$ for a range of lattice thicknesses $k$ at fixed incidence angle $\theta=45^{\circ}$ and as a function of $\theta$ for a range of $p$ values at fixed lattice thickness $k=50$.

As can be seen from Fig. 5, with increasing lattice thickness $k$ the maximum of the relative error becomes larger and its position moves closer to $p=1$. As shown in Appendix D, for all lattices with $k>2$ the position, $p_{\max }$, of the maximum error can be approximated by

$$
p_{\max } \approx \sqrt[1+\tan \theta]{\frac{k-2 \sqrt{k-1}}{k-2}}
$$

which is larger than the percolation threshold $p_{c} \approx 0.59275$ for all lattices $(k>2)$ and incidence angles $\theta \geq 70^{\circ}$. Similarly, 


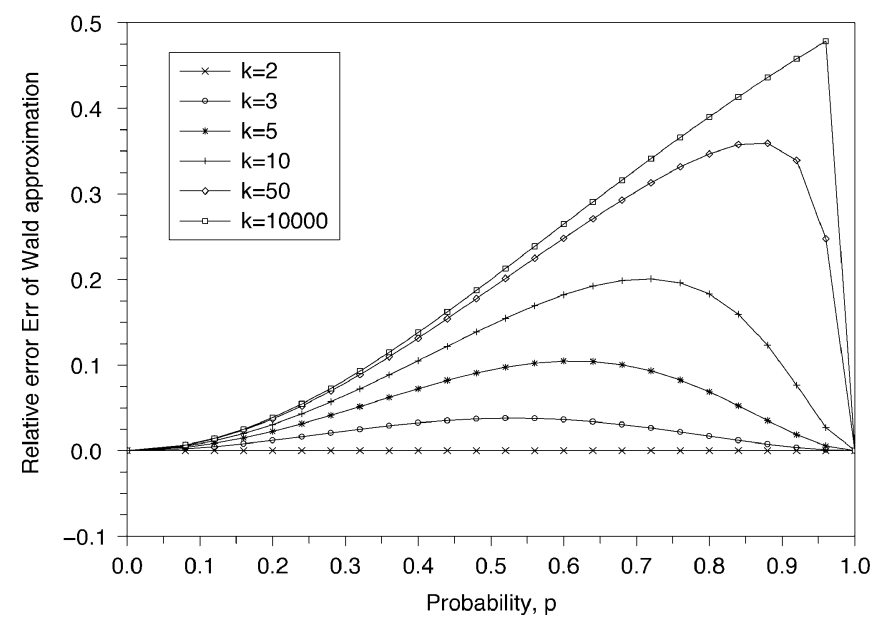

Fig. 5. Relative error, Err (19), of the Wald approximation as a function of probability $p$ and lattice thickness $k$ for incidence angle $\theta=45^{\circ}$.

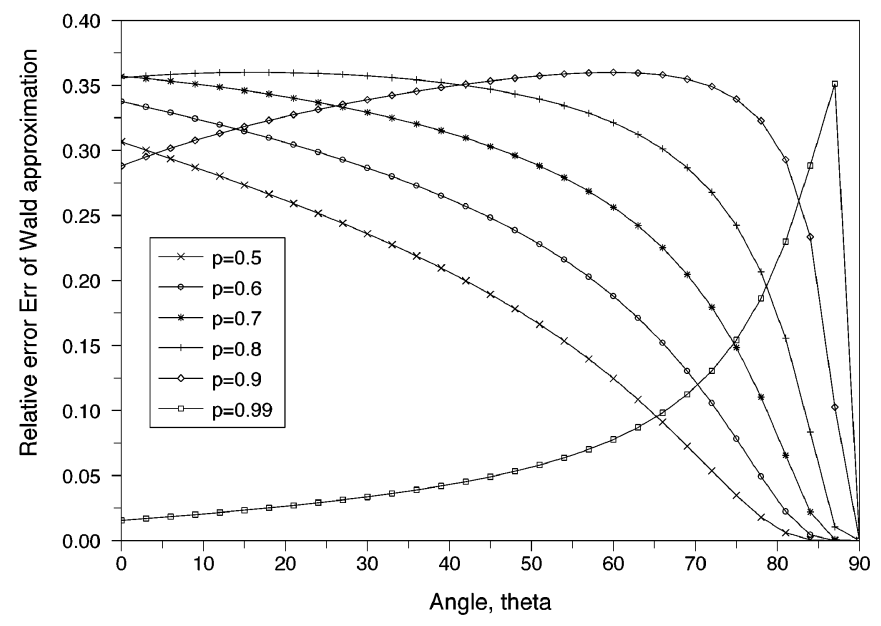

Fig. 6. Relative error, Err (19), of the Wald approximation as a function of incidence angle $\theta$ and probability $p$ for lattice thickness $k=50$.

the relative error is maximal in the area of interest $\left(p>p_{c}\right)$, for instance, when $k>5$ for $\theta \approx 45^{\circ}, k>12$ for $\theta \approx 10^{\circ}$ or $k>16$ for $\theta \approx 0^{\circ}$. At $p=p_{\max }$ the relative error in (19) has the value

$$
\operatorname{Err}_{\max }=\frac{k-2}{2 k} p_{\max }^{1+\tan \theta}=\frac{k-2 \sqrt{k-1}}{2 k}
$$

which, for thick lattices $(k \rightarrow \infty)$, approaches $50 \%$. The maximum relative error, (21), is depicted as a function of lattice thickness $k$ in Fig. 7.

\section{Physical Interpretation of Results}

The probability $Q_{\infty}(k)$ is not as abstract as it appears at first sight. It is useful in the determination of the expectation value for the energy levels at receivers inside a random medium, such as an urban environment. We can assume that the penetration probability $Q_{\infty}(k)$ is equivalent to the probability of the ray's energy being detected at a receiver that is positioned at $k$ and has an energy detection threshold $E_{\min }$, i.e.,

$$
\operatorname{Pr}\left(E(k) \geq E_{\min }\right) \equiv Q_{\infty}(k) .
$$

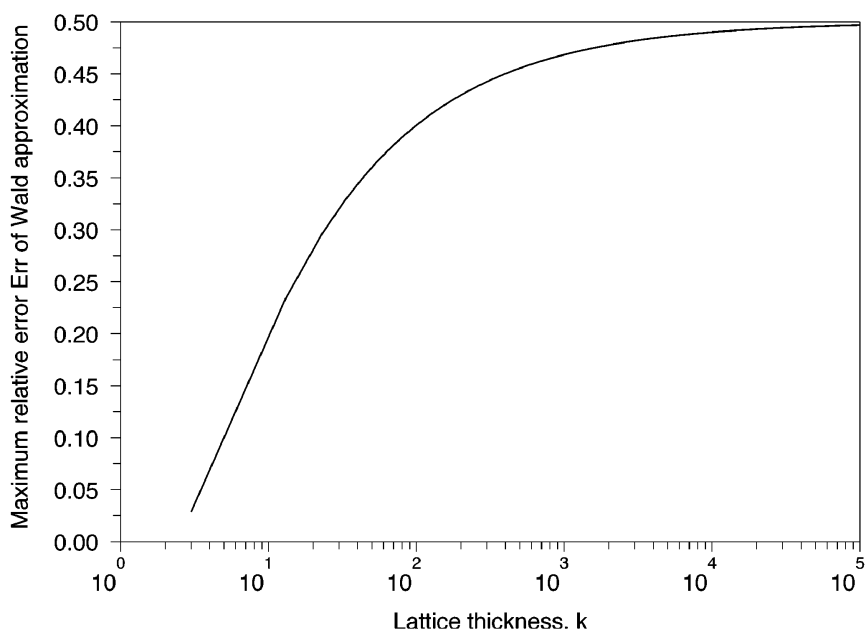

Fig. 7. Approximated maximum relative error, $\operatorname{Err}_{\max }$ in (21), of the Wald approximation as a function of lattice thickness $k$.

Then the conditional expectation of the energy measured at the receiver in $k$ is given by [10]

$$
\bar{E}(k)=\frac{1}{2} Q_{\infty}(k)\left(E_{\max }^{2}-E_{\min }^{2}\right)
$$

where the maximum $E_{\max }$ can be determined from the energy levels in the receiver under LOS conditions. As the expression $(1 / 2)\left(E_{\max }^{2}-E_{\min }^{2}\right)$ is merely a constant, $Q_{\infty}(k)$ essentially describes the dependence of $\bar{E}(k)$ on $k$ and $\theta$. Some work has been published that discusses the experimental validation of (23), [25]-[27].

Note that $Q_{\infty}(k)$ and thus $\bar{E}(k)$ behave like $(c / k)$ for large $k$ with $c=2 p p_{e} / q_{e}$ being a constant that encapsulates characteristics of the (random) medium. This implies that the power of radiation propagating through a lattice of random lossless scatterers decreases with $1 / R$ with $R=a k$ being the distance of a cell from the lattice boundary (i.e., the city border in an urban area). ${ }^{6}$ Considering that a ray ensemble (or a plane wave) does not lose any energy in a vacuum, the $1 / R$-power decrease in $\bar{E}(k)$ is a mere result of lossless scattering inside the lattice. It describes the effect of the random medium (or city) topology on the propagation of the electromagnetic rays.

\section{CONCLUSION}

It has been demonstrated by a number of authors that the application of percolation theory is a suitable starting point for investigating path losses that arise from ray reflections in random media such as urban environments. Percolation theory allows for the formulation of a deterministic propagation model that is based on very few (physical) parameters, and it is this simplicity that facilitates the exploration of concepts, such as the imprinting of urban topology on electromagnetic propagation characteristics. Within the approach a range of tools and methodologies are available, and in some circumstances it is even possible to find analytical solutions. Amongst these tools

${ }^{6} \mathrm{Here}$ the constant $a$ is the lattice spacing, i.e., the distance between the centres of two neighboring lattice cells. For instance, if an urban environment was approximated by the two-dimensional random medium, then $a$ describes the average width of a building. 
are random walk models, which can be analyzed by employing, for instance, the theories of Markov chains or martingales.

In this paper we calculated the probability that an ensemble of (plane-wave) rays reaches a certain depth in a lattice of randomly distributed lossless scatterers under the assumption of independently identically distributed (iid) ray reflections. This probability is closely linked to the conditional expectation of the energy measured in a receiver placed inside the random medium. We derived an explicit expression for the $n$th power of the Markov transition matrix in the limit of infinite reflections, $n \rightarrow \infty$, and proved that both the theories of Markov chains and martingales lead to the same analytical result. We also discussed the accuracy of the commonly used Wald approximation and showed that it can underestimate the penetration probability by up to $50 \%$ even in the domain of scatterer occupancy probabilities $q$ below the percolation threshold $q_{c} \approx 0.40725$ which is the domain of relevance for this problem.

When formulating ray propagation in random media as a twodimensional percolation lattice problem and analyzing it with probabilistic methods, a number of approximations are being made. Firstly, reducing the dimensionality of the problem infers that the model is only applicable when the main propagation is a lateral one. Amongst others, this is the case when a) the transmitter is a far-external source, i.e., so far away from the lattice that the scatterers' heights are negligible; $b$ ) the scatterers-such as buildings in an urban environment-are much higher than the transmitter; or c) transmitters and receivers are located at the same level, such as ultrawideband radios positioned in two-dimensional logistics or security sensor networks. Secondly, describing signal propagation in terms of classical ray optics ignores wave phenomena, in particular diffraction at the sharp edges of obstacles-such as buildings in a city. Thirdly, replacing random media with percolation lattices that consist of "black cells" (lossless scatterers) and "white cell" (vacuum) discounts many effects including attenuation and refraction. Fourthly, the probabilistic approach to solving the percolation-theory based propagation problem is inaccurate in situations when the ensemble average of the scatterer distribution in the lattice is an inappropriate representation of the actual physical process. For example, vertically impinging rays do not see a "gray" lattice but only penetrate to a certain lattice level if and only if all the levels they pass through are "white." Finally, there are assumptions within the probabilistic approach that help make the problem more tractable, e.g., in the case of the work presented here, the simplification was made, that ray reflections are iid.

While this series of approximations may invite criticism, it should be noted that the approach is extendable. In particular ray attenuation and refraction can be included, and the discrepancies between ensemble averaging and percolation simulations can be analysed in order to enhance the applicability of the model. To achieve the latter it is desirable to formulate, and potentially solve, the probabilistic approach exactly, i.e., without resorting to any approximation. In particular, it has been noted [19] that the assumption of iid ray reflections leads to considerable differences between model predictions and field measurements when the signal transmitter is placed inside the lattice. This is mainly due to the fact that the assumption of iid ray reflections results in an inaccurate angle dependence of the calculated probabilities. Hence predictions for phenomena that require the superposition of plane waves, for instance to generate spherical waves, can only be described unsatisfactorily.

As indicated throughout this paper we have managed to generalize the model to allow for an accurate description of ray incidence angles that differ from $45^{\circ}$. This approach is still probabilistic and a generalization of the Markov approach of [9]. It leads to a more accurate description of penetration depths and thus overcomes some of the limitations of the model discussed in this paper and in [19]. We will report on this new approach to percolation-based propagation in random media in [20].

\section{APPENDIX A}

\section{CALCUlation OF EIGENVECTORS $\vec{\omega}_{0}$ AND $\vec{\omega}_{k}$}

The $l$ th component, $\left(M_{k} \vec{\omega}\right)(l)$, of the vector resulting from multiplying matrix $M_{k}$ in (7) and an eigenvector $\vec{\omega}$ of eigenvalue $\lambda=1$ is given by

$$
\begin{aligned}
& \left(M_{k} \vec{\omega}\right)(l)=\frac{1}{2}\left\{p_{e}^{l} \omega(0)+p_{e}^{k-l} \omega(k)\right. \\
& \left.+q_{e} \sum_{m=0}^{l-1} \omega(l-m) p_{e}^{m}+q_{e} \sum_{m=0}^{k-l-1} \omega(l+m) p_{e}^{m}\right\}
\end{aligned}
$$

for $l \neq 0$ and $l \neq k$, and

$$
\left(M_{k} \vec{\omega}\right)(l)=\left\{\begin{array}{lll}
\omega(0) & \text { for } \quad l=0 \\
\omega(k) & \text { for } & l=k
\end{array} .\right.
$$

With $q_{e} \sum_{m=0}^{j-1} p_{e}^{m}=1-p_{e}^{j}$ it is straightforward to show that

$$
p_{e}^{l}+p_{e}^{k-l}+q_{e} \sum_{m=0}^{l-1} p_{e}^{m}+q_{e} \sum_{m=0}^{k-l-1} p_{e}^{m}=2 .
$$

Noting that $\omega(l) \equiv\left(M_{k} \vec{\omega}\right)(l)$ is the equation determining the eigenvector of $\lambda=1$ it follows that

$$
\vec{\omega}_{0}^{T}=\frac{1}{\sqrt{k+1}}(1,1, \ldots, 1)
$$

is one of the (normalized) $\lambda=1$ eigenvectors of $M_{k}$. We could have guessed this result because $M_{k}(1,1, \ldots, 1)^{T}=$ $(1,1, \ldots, 1)^{T}$ corresponds to $\sum_{j=0}^{k} p_{i j}=1 \forall i \in\{0,1, \ldots, k\}$, i.e., the fact that no attenuation (or amplification) of the ray occurs when reflected inside the lattice.

In order to determine the second eigenvector of $\lambda=1$ we introduce the vector $\vec{x}$ with components $x(l) \equiv \omega(l)-\omega(k-l)$. By subtracting (24) with $l \rightarrow k-l$ from itself we get

$$
\begin{aligned}
x(l)= & \frac{1}{2}\left(p_{e}^{l-1}-p_{e}^{k-l-1}\right) x(0) \\
& +\frac{q_{e}}{2}\left\{\sum_{m=1}^{l-1} x(l-m) p_{e}^{m-1}+\sum_{m=1}^{k-l-1} x(l+m) p_{e}^{m-1}\right\} .
\end{aligned}
$$

Without loss of generality we can restrict our further calculations to the case $l<(k / 2)$ noting that $x_{k / 2}=0$ for even $k$. 
Extracting from the last sum in (27) the term proportional to $x_{k-l}$ and using $x_{k-l}=-x_{l}$ yields

$$
\begin{aligned}
x(l)= & \frac{1}{2+q_{e} p_{e}^{k-2 l-1}}\left(p_{e}^{l-1}-p_{e}^{k-l-1}\right) x(0) \\
& +q_{e}\left[\sum_{m=1}^{l-1} x(l-m) p_{e}^{m-1}\right. \\
& +\sum_{m=1}^{k-2 l-1} x(l+m) p_{e}^{m-1} \\
& \left.+\sum_{m=k-2 l+1}^{k-l-1} x(l+m) p_{e}^{m-1}\right] .
\end{aligned}
$$

We can simplify the right side of this equation by replacing $x_{j}$ with $x_{k-j}$ in all terms in which $j \geq k / 2$. After some algebraic manipulations we obtain

$$
\begin{aligned}
\left(2+q_{e} p_{e}^{k-2 l-1}\right) x(l) & =\left(p_{e}^{l-1}-p_{e}^{k-l-1}\right) x(0) \\
& +q_{e} \sum_{m=1}^{l-1} x(m)\left(p_{e}^{l-m-1}-p_{e}^{k-l-m-1}\right) \\
& +q_{e} \sum_{m=l+1}^{\left[\frac{k}{2}\right]-} x(m)\left(p_{e}^{-l+m-1}-p_{e}^{k-l-m-1}\right) .
\end{aligned}
$$

where we have introduced

$$
\left[\frac{k}{2}\right]_{-} \equiv \begin{cases}\frac{k}{2} & \text { for } k \text { even } \\ \frac{k-1}{2} & \text { for } k \text { odd }\end{cases}
$$

Subtracting (28) with $l \rightarrow l+1$ from itself leads to

$$
\begin{aligned}
& \left(2+q_{e} p_{e}^{k-2 l-1}\right) x(l) \\
& -\left(2+q_{e} p_{e}^{k-2 l-3}\right) x(l+1) \\
& =\left(p_{e}^{l-1}-p_{e}^{l+1}\right) x(0) \\
& \quad+q_{e} \sum_{m=1}^{l-1} x(m)\left(p_{e}^{l-m-1}-p_{e}^{l-m+1}\right) \\
& \quad+q_{e}\left(1-p_{e}^{k-2 l-2}\right)\left(x(l+1)-p_{e} x(l)\right)
\end{aligned}
$$

Hence, for $1 \leq l \leq[(k / 2)]_{-}$

$$
\begin{aligned}
x(l+1)= & \frac{1}{2 p_{e}+q_{e}}\left\{\left(2+q_{e} p_{e}\right) x(l)\right. \\
& \left.-\left(1-p_{e}^{2}\right)\left[p_{e}^{l-1} x(0)+q_{e} \sum_{m=1}^{l-1} p_{e}^{l-m-1} x(m)\right]\right\} \\
= & \left(1+q_{e}\right) x(l)-q_{e}^{2} \sum_{m=1}^{l-1} p_{e}^{l-m-1} x(m)-q_{e} p_{e}^{l-1} x(0) .
\end{aligned}
$$

Thus it follows that

$$
x(l+1)=\left(1+l q_{e}\right) x(1)-l q_{e} x(0)
$$

as can be proved by induction:

1) Given (31) we get for $l=1$ :

$$
\begin{aligned}
x(2) & =\left(1+q_{e}\right) x(1)-q_{e}^{2} \sum_{m=1}^{0} p_{e}^{l-m-1} x(m)-q_{e} x(0) \\
& =\left(1+q_{e}\right) x(1)-q_{e} x(0)
\end{aligned}
$$

which shows that (32) is true for $l=1$.

2) Now assume that (32) is true for all $l \leq n$, which also implies that

$$
x(l+1)-x(l) \equiv q_{e}(x(1)-x(0)) \forall l \leq n .
$$

From (31) it then follows that:

$$
\begin{aligned}
x(n+ & 2)-x(n+1) \\
= & \left(1+q_{e}\right)[x(n+1)-x(n)] \\
& -q_{e}\left(p_{e}^{n}-p_{e}^{n-1}\right) x(0) \\
& -q_{e}^{2}\left[\sum_{m=1}^{n} p_{e}^{n-m} x(m)-\sum_{m=1}^{n-1} p_{e}^{n-m-1} x(m)\right] \\
= & \left(1+q_{e}\right) q_{e}[x(1)-x(0)]+q_{e}^{2} p_{e}^{n-1} x(0) \\
& -q_{e}^{2}\left[\sum_{m=2}^{n} p_{e}^{n-m}\{x(m)-x(m-1)\}+p_{e}^{n-1} x(1)\right] \\
= & \left(1+q_{e}\right) q_{e}[x(1)-x(0)]+q_{e}^{2} p_{e}^{n-1} x(0) \\
& -q_{e}^{3}[x(1)-x(0)] \sum_{m=2}^{n} p_{e}^{n-m}-q_{e}^{2} p_{e}^{n-1} x(1) \\
= & \left(1+q_{e}-q_{e} p_{e}^{n-1}-q_{e}^{2} \frac{1-p_{e}^{n-1}}{q_{e}}\right) q_{e}[x(1)-x(0)] \\
= & q_{e}[x(1)-x(0)]
\end{aligned}
$$

QED.

Inserting (32) into (28) and some algebraic manipulations lead to

$$
\begin{aligned}
\frac{x(1)}{x(0)} & =\frac{\left.p^{\left[\frac{k}{2}\right]}\right]_{-}-p_{e}^{\left[\frac{k}{2}\right]--2}+q_{e}\left[\frac{k}{2}\right]_{-}\left(p^{\left[\frac{k}{2}\right]--1}+p^{\left[\frac{k}{2}\right]--2}\right)}{\left(1+p_{e}\right) p_{e}^{\left[\frac{k}{2}\right]--1}+q_{e}\left[\frac{k}{2}\right]_{-}\left(p_{e}^{\left[\frac{k}{2}\right]--1}+p_{e}^{\left[\frac{k}{2}\right]--2}\right)} \\
& =\frac{q_{e}(k-2)}{q_{e} k+2 p_{e}} .
\end{aligned}
$$

Thus for $1 \leq l \leq[(k / 2)]$ - we get

$$
x(l)=\frac{q_{e}(k-2 l)}{q_{e} k+2 p_{e}} x(0) .
$$

Using the relation $x(k-l)=-x(k)$ it can be shown that this equation is also true for $[(k / 2)]_{-} \leq l \leq k$. Thus we finally obtain

$$
\vec{\omega}_{k}=Z^{-1}\left(\begin{array}{c}
2+q_{e}(k-2) \\
q_{e}(k-2) \\
q_{e}(k-4) \\
\vdots \\
q_{e}(k-2(k-1)) \\
-2-q_{e}(k-2)
\end{array}\right)
$$


for the second (normalized) eigenvector of eigenvalue $\lambda=1$. Here, the normalization constant is given by

$$
Z=\sqrt{2\left[2+q_{e}(k-2)\right]^{2}+\frac{1}{3} q_{e}^{2} k(k-1)(k-2)} .
$$

\section{APPENDIX B \\ FIRST AND LAST ROW OF $\Lambda_{k}^{-1}$}

In this Appendix we describe how to calculate the first and last row of the inverse to the spectral matrix $\Lambda_{k}$ which, as mentioned after (11), we need in order to determine the solution of (5). From the eigenvalue equation (9) it follows that:

$$
\left(M_{k}^{-1}\right)^{T}\left(\Lambda_{k}^{-1}\right)^{T}=\left(\Lambda_{k}^{-1}\right)^{T} \operatorname{diag}\left(\frac{1}{\lambda_{0}}, \frac{1}{\lambda_{1}}, \ldots, \frac{1}{\lambda_{k}}\right)
$$

which shows that the first and last row of the inverse to $\Lambda_{k}$ are right eigenvectors of $\left(M_{k}^{-1}\right)^{T}$ to eigenvalue $(1) /\left(\lambda_{0}\right)=$ $(1) /\left(\lambda_{k}\right)=1$.

Given the definition of matrix $M_{k}$, (7), it is straightforward to show that $M_{k}^{-1}$ must have the following form:

$$
M_{k}^{-1} \equiv\left(\begin{array}{cccc}
1 & 0 & \cdots & 0 \\
m_{10} & m_{11} & \cdots & m_{1 k} \\
m_{20} & m_{21} & \cdots & m_{2 k} \\
\vdots & \vdots & \vdots & \vdots \\
m_{(k-1) 0} & m_{(k-1) 1} & \cdots & m_{(k-1) k} \\
0 & \cdots & 0 & 1
\end{array}\right)
$$

Thus $\vec{e}_{0}=(1,0, \ldots, 0)^{T}$ and $\vec{e}_{k}=(0, \ldots, 0,1)^{T}$ are eigenvectors of $\left(M_{k}^{-1}\right)^{T}$ with eigenvalue 1 . From this it follows that the linear combinations $\vec{\beta}_{0}=\beta_{00} \vec{e}_{0}+\beta_{0 k} \vec{e}_{k}$ and $\vec{\beta}_{k}=\beta_{k 0} \vec{e}_{0}+$ $\beta_{k k} \vec{e}_{k}$ are the two eigenvectors of $\left(\Lambda_{k}^{-1}\right)^{T} \equiv\left(\vec{\beta}_{0}, \vec{\beta}_{1}, \ldots, \vec{\beta}_{k}\right)$ that we need to evaluate.

From

$$
I_{k+1}=\Lambda_{k}^{-1} \Lambda_{k}=\left(\begin{array}{ccc}
\vec{\beta}_{0}^{T} \vec{\omega}_{0} & \cdots & \vec{\beta}_{0}^{T} \vec{\omega}_{k} \\
\vdots & \ddots & \vdots \\
\vec{\beta}_{k}^{T} \vec{\omega}_{0} & \cdots & \vec{\beta}_{k}^{T} \vec{\omega}_{k}
\end{array}\right)
$$

we get the system of equations $\vec{\beta}_{0}^{T} \vec{\omega}_{0}=1, \vec{\beta}_{0}^{T} \vec{\omega}_{k}=0, \vec{\beta}_{k}^{T} \vec{\omega}_{0}=$ 0 and $\vec{\beta}_{k}^{T} \vec{\omega}_{k}=1$, which can easily be solved, given $\vec{\omega}_{0}$ and $\vec{\omega}_{k}$ in (26) and (35), respectively. The solutions are

$$
\begin{aligned}
& \beta_{00}=\beta_{0 k}=\frac{1}{2} \sqrt{k+1} \\
& \beta_{k 0}=-\beta_{k k}=\frac{Z}{2\left(2 p_{e}+k q_{e}\right)}
\end{aligned}
$$

with $Z$ given in (36).

\section{APPENDIX C}

PROOF OF (13)

When inserting the expression 12 for $M_{k}^{\infty}$ into (5) one obtains:

$$
\vec{Q}_{\infty}^{T}=\left(1-p p_{e}^{k-1}-Y, 0, \ldots, 0, p p_{e}^{k-1}+Y\right)
$$

with

$$
Y=\frac{p q_{e}}{2 p_{e}+k q_{e}} \sum_{s=1}^{k-1}\left(1+(s-1) q_{e}\right) p_{e}^{s-1} .
$$

Using

$$
\begin{aligned}
\sum_{s=1}^{k-1} p_{e}^{s-1} & =\frac{1}{q_{e}}\left(1-p_{e}^{k-1}\right) \\
\sum_{s=1}^{k-1}(s-1) p_{e}^{s-1} & =\frac{p_{e}}{q_{e}^{2}}\left(1-p_{e}^{k-1}\right)-(k-1) \frac{p_{e}^{k-1}}{q_{e}}
\end{aligned}
$$

we get

$$
Y=p \frac{2 p_{e}+q_{e}}{2 p_{e}+k q_{e}}-p p_{e}^{k-1}
$$

and hence,

$$
\vec{Q}_{\infty}^{T}=\left(1-p \frac{2 p_{e}+q_{e}}{2 p_{e}+k q_{e}}, 0, \ldots, 0, p \frac{2 p_{e}+q_{e}}{2 p_{e}+k q_{e}}\right)
$$

which is (13).

\section{APPENDIX D \\ PROOF OF (20)}

Given the function Err we can determine the maximum position $p_{\max }$ by finding the zeros of the $p_{e}$-differentiated (19)

$$
0=(k-2) p_{e}^{2}-2 k p_{e}+k-2+O\left(p_{e}^{k-1}\right) .
$$

Neglecting terms of order $O\left(p_{e}^{k-1}\right)$ (which is approximately true for $k>2$ ) we obtain (20) as the only solution with $p \leq 1$.

\section{ACKNOWLEDGMENT}

The authors would like to thank Dr. C. Coleman from the School of Electrical and Electronic Engineering, University of Adelaide, Australia, for useful discussions.

\section{REFERENCES}

[1] H. Bertoni, W. Honcharenko, K. Kim, L. Macel, and H. Xia, "Uhf propagation prediction for wireless personal communications," Proc. IEEE, vol. 82, no. 9, pp. 1333-1359, 1994.

[2] M. Iskander and Z. Yun, "Propagation prediction models for wireless communication systems," IEEE Trans. Microw. Theory Tech., vol. 50, no. 3, pp. 662-673, 2002.

[3] T. Sarkar, Z. Ji, K. Kim, A. Medouri, and M. Salazar-Palma, "A survey of various propagation models for mobile communication," IEEE Trans. Antennas Propag., vol. 45, no. 3, pp. 51-82, 2003. 
[4] T. Okumura, E. Ohmori, and F. K. , "Field strength and its variability in VHF and UHF land mobile service," Rev. Elect. Commun. Lab., vol. 16, no. 9-10, pp. 825-873, 1968.

[5] E. Damosso, "Digital Mobile Radio: COST 231 View on the Evolution Towards 3rd Generation Systems," Bruxelles: Final Rep. COST 231 Project 1998, European Commission.

[6] M. Hata, "Empirical formula for propagation loss in land mobile radio service," IEEE Trans.. Veh. Technol., vol. VT-29, no. 3, pp. 317-325, 1980.

[7] F. Ikegami, S. Yoshida, T. Takeuchi, and M. Umeira, "Propagation factors controlling mean field strength on urban streets," IEEE Trans. Antennas Propag., vol. AP-26, no. 8, 1984.

[8] J. Walfisch and H. Bertoni, "A theoretical model of UHF propagation in urban environments," IEEE Trans. Antennas Propag., vol. AP-36, no. 12 , pp. $1788-1796,1988$.

[9] G. Franceschetti, S. Marano, and F. Palmieri, "Propagation without wave equation toward an urban area model," IEEE Trans. Antennas Propag., vol. 47, no. 9, pp. 1393-1404, 1999.

[10] B. Di Chiara, R. Sorrentino, M. Strappini, and L. Tarricone, "Electromagnetic (EM) prediction in complex scenarios: Advances in percolative modelling," in Proc. Int. Symp. on Electromagnetic Theory, Pisa, Italy, May 23-27, 2004, pp. 561-563, 2004.

[11] S. R. Broadbent and J. M. Hammersley, "Percolation processes. I. crystals and mazes," Proc. Cambridge Philos. Soc., vol. 53, pp. 629-641, 1957.

[12] G. Mackay and N. Jan, "Forest fires as critical phenomena," J. Phys. A-Math. Gen., vol. 17, pp. L757-L760, 1984.

[13] D. Stauffer, A. Coniglio, and M. Adam, "Gelation and critical phenomena," Adv. Polym. Sci., vol. 44, pp. 103-158, 1982.

[14] B. I. Shklovskii and A. L. Efros, Electronic Properties of Doped Semiconductors. Heidelberg, Germany: Springer, 1984.

[15] H. Kesten, Percolation Theory for Mathematicians. New York: Birkhauser, 1982.

[16] D. Stauffer, Introduction to Percolation Theory.. London, U.K.: Taylor Francis, 1985.

[17] A. Martini, M. Franceschetti, and A. Massa, "Ray propagation in non-uniform random lattices," J. Opt. Soc. Am. A, vol. 23, no. 9, pp. 2251-2261, 2006.

[18] Gantmacher, Applications of the Theory of Matrices.. New York: Interscience, 1959, (English translation).

[19] G. Franceschetti, S. Marano, and F. Palmieri, "Percolation model for urban area propagation: Results and open problems," in Proc. IEEE Antennas and Propagation Symp., San Antonio, TX, Jun. 16-21, 2002, pp. 224-227, 2002.

[20] A. Bender and T. Rainsford, "Inhomogeneous Markov approach to percolation theory based propagation in random media," IEEE Trans. Antennas Propag., accepted for publication.

[21] R. G. Gallager, Discrete Stochastic Processes, 3rd ed. Boston, MA: Luver Academic, 1999.

[22] W. Feller, An Introduction to Probability Theory and Its Application, 3rd ed. New York: Wiley, 1968, vol. I.

[23] W. Feller, An introduction to Probability Theory and Its Application.. New York: Wiley, 1967, vol. II.

[24] S. Marano, F. Palmieri, and G. Franceschetti, "Statistical characterization of ray propagation in a random lattice," J. Opt. Soc. Am. A, vol. 16, no. 10, pp. 2459-2464, 1999.
[25] M. Montepeloso, R. Sorrentino, M. Strappini, and L. Tarricone, "Nowave-equation radiopropagation models: Experiences with the percolation theory," presented at the IEEE AP-S Int. Symp. and USNC/CNC/URSI National Radio Science Meeting, Columbus, $\mathrm{OH}$, Jun. 22-27, 2003, 2003.

[26] M. Angelucci, B. Di Chiara, R. Sorrentino, M. Strappini, and L. Tarricone, "Genetic optimization of radiobase-station sizing and location using a GIS-based framework: Experimental validation," presented at the IEEE AP-S Int. Symp. and USNC/CNC/URSI national radio science meeting, Columbus, OH, Jun. 22-27, 2003, 2003.

[27] M. Angelucci, B. Di Chiara, M. Montepeloso, R. Sorrentino, M. Strappini, and L. Tarricone, "Conventional radiopropagation models and no-wave equation approaches: An application of the percolation theory," in Proc. 33rd Eur. Microwave Conf., Munich, Germany, Oct. 7-9, 2003, vol. 3, pp. 1163-1166, 2003.

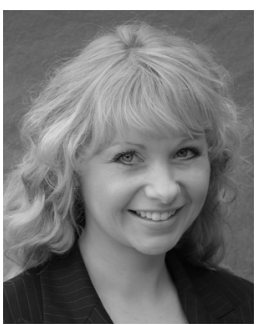

Tamath Rainsford received the Ph.D. degree in mathematical physics from the University of Adelaide, Australia, in 2000.

She is a Lecturer and Research Fellow in the School of Electrical and Electronic Engineering, University of Adelaide and is a member of the Centre for Biomedical Engineering at the same university. From 1999 to 2002, she held a Postdoctoral Research Fellow position in the Department of Human Physiology, School of Medicine, Flinders University, Australia, in the area of neurogastroenterology and from 2003 to 2004 she held the same position at the School of Molecular and Biomedical Science, University of Adelaide, in the fields of insect vision and motion detection. Her research interests include biomedical spectroscopy, imaging and pattern classification including terahertz biosensing, electromagnetic wave propagation especially of terahertz in human tissue, implementation of bioinspired approaches to artificial vision systems in VLSI and neural encoding, networks and noise of small neural systems.

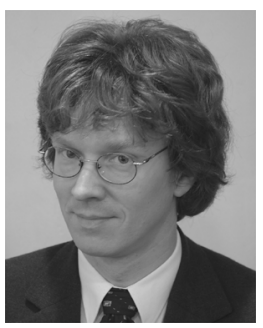

Axel Bender received the Ph.D. degree in theoretical particle physics from Tübingen University, Germany, in 1994.

Currently, he is a Senior Research Scientist in the Land Operations Division, Defence Science and Technology Organisation (DSTO), Australia. From 1994 to 1996 , he undertook research in non-perturbative quantum field theories at the Argonne National Laboratory, and from 1996 to 1998 , held a Postdoctoral Research Fellow position with the Special Research Centre for the Subatomic Structure of Matter, University of Adelaide, Australia. From 1998 to 2003, he was a Statistics Research Officer at the National Centre for Vocational Education Research and joined the DSTO in 2003. Since then he has been working on supply chain management problems, complex systems, military experimentation and science and technology support to defence capability development projects. 